\title{
Autoselection Phenomenon in the Normal Cell Clone Undergoing Differentiation: From Cell Population Heterogeneity to Cancer Phenotype via Nonmutational Changes
}

\author{
GALINA I. SOLYANIK $^{\mathrm{a}, *}$ and ALEXANDER A. SERIKOV
}

${ }^{a}$ Institute of Experimental Pathology, Oncology and Radiobiology, Kiev 252022, Ukraine; ${ }^{b}$ Bogolyubov Institute for Theoretical Physics and Scientific Research Centre of Quantum Medicine «Vidhuk», Kiev 252143, Ukraine

(Received 22 November 1998; In final form 9 August 1999)

\begin{abstract}
The problem of regulation of the proliferation-differentiation processes is analysed in the framework of the model of unipotential cell clone dynamics, assuming two alternative ways (scenarios) of an every new-born cell development, which in the long run define correspondingly the proliferating or differentiated cell status. The scenario choice is then supposed to be a random event influenced by both the inherent mitotic activity of the cell and the differentiation factors - special messengers synthesized by the differentiated cells. Differentiation factors being accepted by every-new born cell can change the way of cell development. Taking into account the clonal heterogeneity it was shown that such mechanism of the regulation of proliferation-differentiation processes can result in the selection of actively proliferating and insensitive with respect to differentiation factors action cells (that is to say, the cells with cancer phenotype). In the framework of investigation the cells with cancer phenotype (which is heritable within the variability of mother-daughter correlations) appears through the series of the permanent nonmutational changes in the proliferating cells by autoselection mechanism retaining for the division and generation of the progeny only that actively proliferating cells which are less sensitive with respect to the action of the differentiation factors. The progression of normal cell clone towards the appearance and increase of the number of the cells with cancer phenotype can be considered as a possible mechanism of carcinogenesis which is alternative to the clonal selection theory of cancer origin.
\end{abstract}

Keywords: Cell population heterogeneity, autoselection phenomenon, carcinogenesis

\section{INTRODUCTION}

The development and functioning of a multicellular organism as well as any of its organ are impossible without regulation of cell division and cell differentiation processes. It is just their mutual regulation, on the one hand, causes step-by-step narrowing of cell potentiality (from omni- and multipotential - to

\footnotetext{
${ }^{*}$ Corresponding Author: E-mail: gis@onconet.kiev.ua
} 
oligo- and unipotential cell states) in the course of the organism formation and, on the other hand, maintains the renewal of cellular composition and spatial arrangement of tissues during the reparation processes in adult organism. Disturbances in the above regulation are then considered to be responsible for a number of pathological processes in organism, in particular, for tumour origination (Hurlin et al., 1995; Enrietto \& Beug, 1994).

At present there are no consensus on the mechanisms of the regulation (control) of cell division/differentiation processes. Thus, some authors give preference to the concept of genetic predetermination of the regulation (self-regulation on an individual cell level), which finds confirmation in the spontaneous differentiation (transdetermination) phenomena. Others choose the specific regulation concept (DiCicco-Bloom et al, 1990; Fuchs, 1990; Milner et al., 1996) assuming the differentiation being caused by cell interaction with each other (mediated via specific inductors - repressors / derepressors) or with the environment (e.g., with the whole embryo).

However, none of the concepts, irrespective of an extent of their experimental verification, are capable of giving a complete solution of the regulation problem (neither from molecular-biological, nor from embryological stand points) till they do not touch the question of interdependence between the proliferation and differentiation processes in the heterogeneous cell population as a whole.

The purpose of the present paper is to show that in the heterogeneous cell population the mechanism of proliferation-differentiation processes based on the inhibition of the proliferating cells by differentiation factors (special messengers synthesized by the differentiated cells) can result in the selection of actively proliferating and insensitive with respect to the action of differentiation factors cells. We called this phenomenon an autoselection. As insensitivity of the cells to the action of differentiation factors is one of the main peculiarity that differ the cancer cell from the normal one the autoselection phenomenon in the heterogeneous normal cell populations undergoing differentiation can be considered as one of a possible mechanism of carcinogenesis.

\section{THE DYNAMICS OF CLONE WITH THE HOMOGENEOUS POOLS OF PROLIFERATING AND DIFFERENTIATED NORMAL CELLS}

In our previous work [Serikov \& Solyanik, 1998] the problem of regulation of the proliferationdifferentiation processes in some tissues of adult organism has been analyzed by stochastic model of cell clone dynamics, assuming two alternative ways (scenarios) of an every new-born cell development, which in the long run define correspondingly the proliferating or differentiated cell status. The scenario choice is then supposed to be a random event influenced by both the inherent mitotic activity of the cell and the differentiation factors (DF) - special messengers synthesized by the differentiated cells. The model has been created under the following assumptions:

- the population (clone) is originated from one and only progenitor cell;

- there are two alternative scenarios of an every new-born cell development: one - leading to its division, another - to differentiation (the scenario choice is presumably ascertained during the presynthetic phase of the cell mitotic cycle);

- all the cells of the clone are unipotential (with the same allowable type of their differentiation);

- cell differentiation is an irreversible act; thus, the proliferating pool restores its loss only by cell division - not by dedifferentiation processes;

- all the proliferating cells are characterized by the same value $\left(T_{c}\right)$ of the mitotic cycle time; thus, all the cells of $n$-th generation come into being at the moment $T_{c}$ counting after the progenitorcell arising, and at any given moment of time the proliferating pool includes the cells of one generation only;

- the life time of the differentiated cells is long as against $T_{c}$, and so, an every cell of the 
kind co-exists with a number of generations of proliferating cells;

- differentiated cells when functioning, synthesize special messengers-the differentiation factors (DF) which being fixed by new-born cells, inhibit their division and induce differentiation;

- the environment maintains a sufficient level of nutrients (both specific and non-specific) to ensure cell viability and the division processes; so, one can neglect cell loss from starvation and so forth.

In the framework of this model the dynamics of the homogeneous normal cell clone in terms of the average values $X(n), Y(n)$ of the numbers of all proliferating cells $X_{n}$ of $n$-th generation, and those that undergo differentiation $Y_{n}$ can be calculated using the following recurrence relations:

$$
\begin{aligned}
\mathrm{X}(\mathrm{n}+1) & =2 \mathbf{w}_{\mathrm{n}} \mathrm{X}(\mathrm{n}) \\
\mathrm{Y}(\mathrm{n}) & =\left(1-\mathbf{w}_{\mathrm{n}}\right) \mathrm{X}(\mathrm{n}), \\
\mathrm{X}(0) & =1, \quad \mathrm{Y}(0)=0, \\
\text { with } \quad \mathrm{w}_{\mathrm{n}} & =\mathbf{w}\left(\mathrm{p}_{\mathrm{n}} ; \boldsymbol{k}\right)=\left[1+\exp \left(\mathrm{p}_{\mathrm{n}}-\boldsymbol{k}\right)\right]^{-1}, \\
\mathrm{p}_{\mathrm{n}} & =\mathrm{q} \mathrm{Y}(\mathrm{n}-1) /[\mathrm{X}(\mathrm{n})+\mathrm{g}]+\mathrm{p}_{\mathrm{n}-1} / 2
\end{aligned}
$$

where $\mathbf{w}_{\mathrm{n}}$ denotes the probabilities of cell division (as a choice of cell development) and depends on (Equation (2)) an inherent mitotic activity of the cell $(\boldsymbol{k})$ and the number of differentiation factors accepted by the cell $\left(p_{n}\right)$. The later is defined by the Equation (3), where parameter $q$ characterizes the number of DF synthesized by every differentiated cell, and parameter $g$ reflects the average life time of the DF.

The values of the parameter $\boldsymbol{k}$ (referred below as IMA-index), ranging from $-\infty$ to $+\infty$, characterize the balance of the intracellular regulators of mitotic activity (to a considering extent genetically predetermined): positive (enhancing mitotic activity of the cell) and negative (on the contrary, inhibiting proliferation and promoting differentiation). The positive regulators could be represented by the products encoded by proto-oncogenes (in particular, by myc-family of the genes), whereas the negative ones - by the products encoded by suppressor genes (such as RB-gene or gene encoding p53-protein) (Green, 1989). The DF fixed by the cell shift the balance of regulators, causing the corresponding reduction of the mitotic activity.

It is worth paying attention that in the framework of the model the cells characterized by high $k$-values and so actively proliferating, at the same time, are the least sensitive to the DF action. It is very important because the insensitivity of the cells with respect to the action of differentiation factors is one of the main peculiarities that differs the cancer cells from the normal one. Thus in the framework of the model the cells with high $k$-values can be considered to be characterized by cancer phenotype.

The results obtained by the calculating of the model (Figure 1) demonstrate a multiplicity of the different asymptotic behaviours of clone development depending on the inherent mitotic activity of the cells. With the increase of the $k$-value the dynamic picture of the changes in the number of proliferating cells becomes nonmonotonic and then goes over into undemped quasiharmonic oscillations (the amplitude and period of which rise together with $k$-value). With further increase of the parameter $k$ the oscillations change into contrasting picture of extended (about several tens of mitotic cycle periods) intervals of quasi-exponential growth of the number of cells altering with those of its sharp (during 2 or 3 mitotic cycle periods) fall. Finally, the asymptotic dynamics of the clone growth is transformed to exponential one.

The computer simulations of the model taking into account the fluctuations of the integer number of proliferating and differentiated cells resulted from the random character of the processes showed (Figure 2) the interesting characteristic peculiarity of the stochastic dynamics of the integer number of cells - the possibility of the irreversible disappearance of the proliferating pool due to differentiation of all new-born cell near the minimum of the (oscillating) system dynamics (Figure $2 \mathrm{~d}$ ). In the framework of the model the probability of the proliferating pool death increases together with $k$-value. 


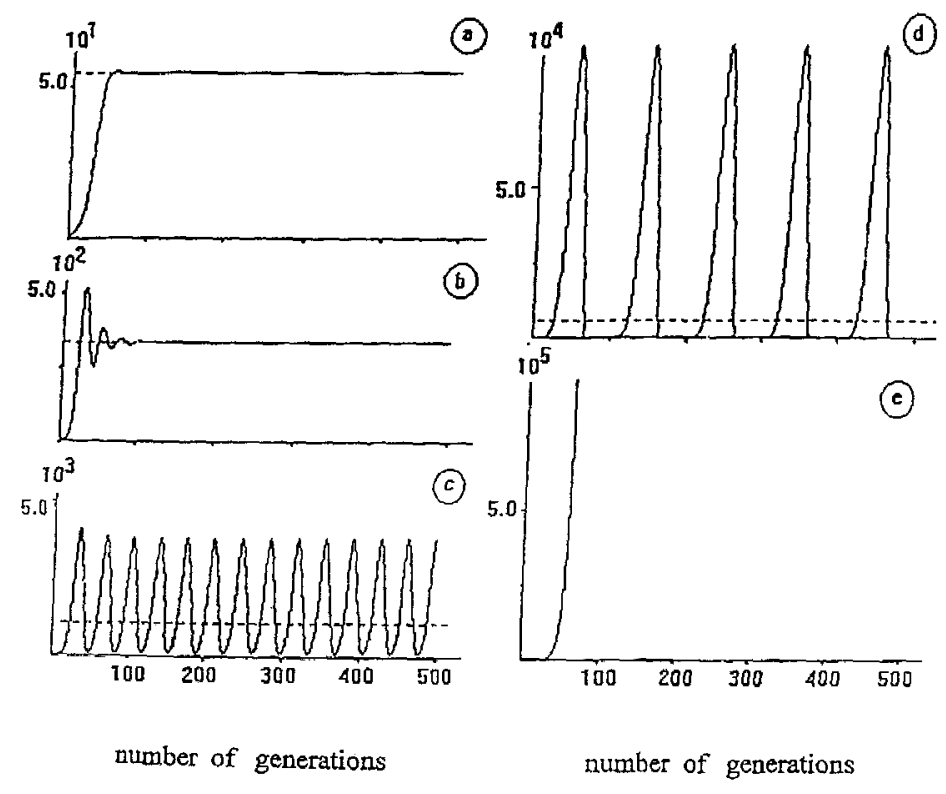

FIGURE 1 Dynamics of homogeneous clone development in terms of the number of proliferating cells per clone (y axis) calculated by the model (1)-(3). a, b, c, d, e corresponds to the clones with different $\mathrm{k}$-values of the inherent mitotic activity of the cells: $0.3,0.7,1.05$, $1.15,1.2$, respectively $(q=1.2, \mathrm{~g}=200)$.

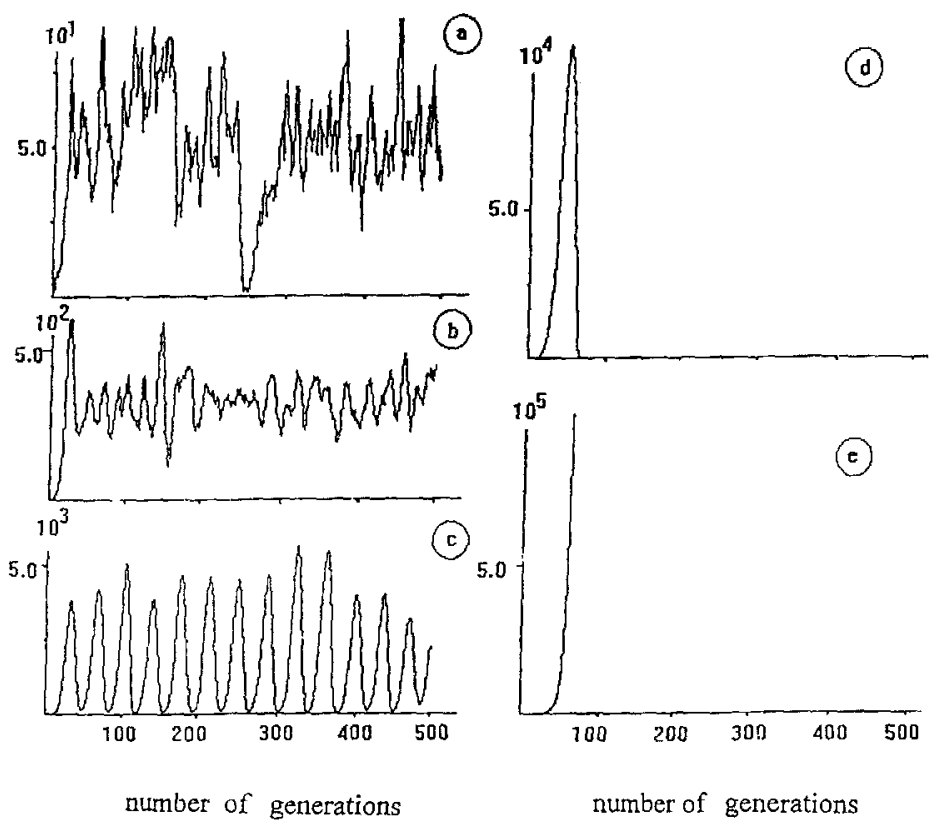

FIGURE 2 Dynamics of homogeneous clone development in terms of the integer number of proliferating cells in a clone ( $y$ axis) simulated by the model (1)-(3) (taking into account the random character of proliferation/differentiation processes). a, b, c, d, e-the same as in the Figure. 1. 


\section{THE INFLUENCE OF CLONAL HETEROGENEITY ON THE DYNAMICS OF THE DEVELOPMENT OF NORMAL CELL POPULATION UNDERGOING DIFFERENTIATION}

One of the main and sufficiently strong assumption of the considered model is the genetic homogeneity of the pools of proliferating and differentiated cells. Meanwhile, the homogeneity of normal cell clones, formerly considered as one of the main principle of cellular biology, has been essentially revised over the last decade. Experimental investigations of a number of cellular characteristics showed that the heterogeneity is inherent to the clones of the normal cells (Axelrod \& Kuczek, 1989) as well as to the cancer one (Heppner, 1984). In this connection in the framework of the above model the development of normal cell clone taking into account its heterogeneity has been considered.

The modification of the model in this case includes the following assumptions (Figure 3):

- The progenitor-cell of the clone belong to the population of genetically identical cells the phenotypic distribution of which with respect to IMA-index is considered to be in a form of Gauss distribution with $K_{\mathrm{p}}$ - as the mean of the (stable!) population and $C_{p}$ - as an average deviation characterizing the extent of population heterogeneity $\left(\boldsymbol{C}_{p}\right.$ is the population heterogeneity parameter);

- For each value $\boldsymbol{K}_{\mathrm{m}}$ of progenitor cell (from the population distribution) the probability density function of the corresponding characteristics for the daughter cells also can be described by Gauss distribution, where $\boldsymbol{C}_{\mathrm{c}}$ determines the stochastic deviation of the daughter values of inherent mitotic activity - clone heterogeneity parameter.

It should be noted that many experimental investigations showed more considerable intrapopulation variation with respect to intraclonal one. Thus, for example, the experimental study of keratocytes of guinea-pig ear epidermis (carried out as early as 1981) showed that intrapopulation variation of interdivision time more than three time greater than that of

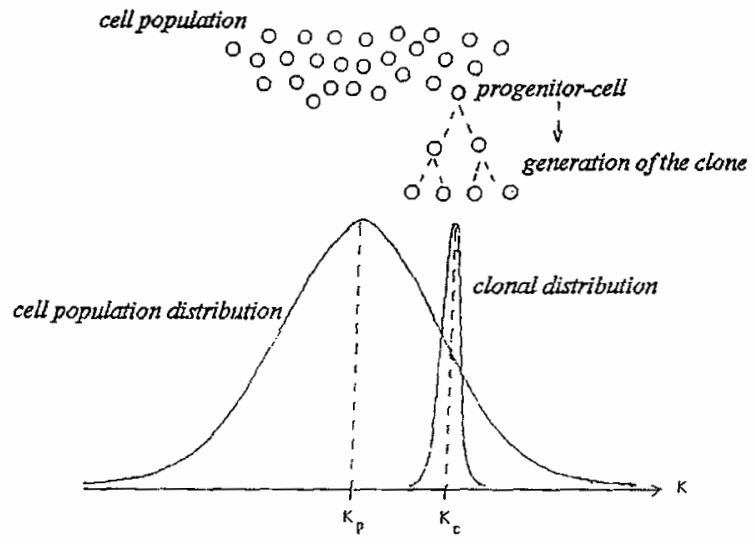

FIGURE 3 Distribution of the stable cell population and the clone originated from this population with respect to the inherent mitotic activity index $(\boldsymbol{K})$ of proliferating cells.

intraclonal (Relay \& Hola, 1981). That is why in our study we analyzed only the situation when $C_{\mathrm{c}}>C_{\mathrm{p}}$.

The computer simulations of the model taking into account the intraclonal and intrapopulation variability (Appendix) showed two different ways of clone development.

The first of them - the regression of progeny to a population mean (Figure 4). In the framework of such behaviour it is seen that the higher the intraclonal variability the more rapid regression toward mean is observed. It worth paying attention also that the dynamics of heterogeneous clone development in this case (Figure 5) is similar to that which has been obtained in the framework of homogeneous model (see Figure 2b).

It should be noted that this type of clone development have their experimental evidence (Gamel \& Axelrod, 1991). Moreover in population dynamics the regression of progeny to a population mean is known as a «law of filial regression》 which has been firstly described and formulated by Galton (1989).

The increase of cell population heterogeneity results in other scenario of clone development. The regression of clone to the mean of the cell population in this case (which also takes place under high intraclonal variability) is changed (with the decrease of clone variability) by the progression toward the high $\boldsymbol{K}$ values (Figure 6). The dynamics of clone demonstrates the successive changes of asymptotic behaviour of the proliferating pool: from the limited 


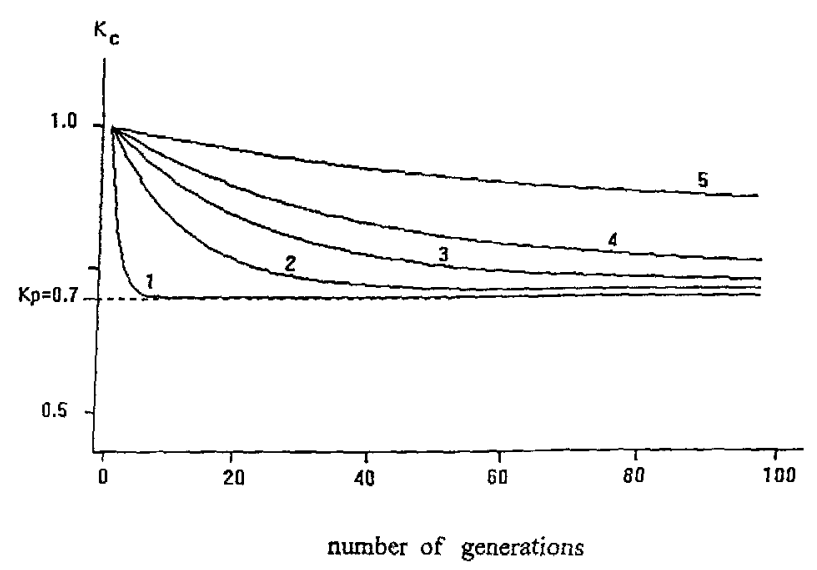

FIGURE 4 Regression of the mean clone value of the mitotic activity index $\boldsymbol{K}_{\mathrm{c}}$ to the mean value $\boldsymbol{K}_{\mathrm{p}}=0.7$ of the stable population. The stochastic deviation of the stable population $C_{p}=20$. Different curves correspond to the different stochastic deviation of the clonal distribution $C_{c}=C_{\mathrm{p}}(1) ; 2 C_{\mathrm{p}}(2) ; 3 C_{\mathrm{p}}(3) ; 4 C_{\mathrm{p}}(4) ; 7 C_{\mathrm{p}}(5)$. The $k$-value of the mitotic activity index of the progenitor cell (in the Figure 4-Figure 8) equals $1, q=1.2, g=200$.

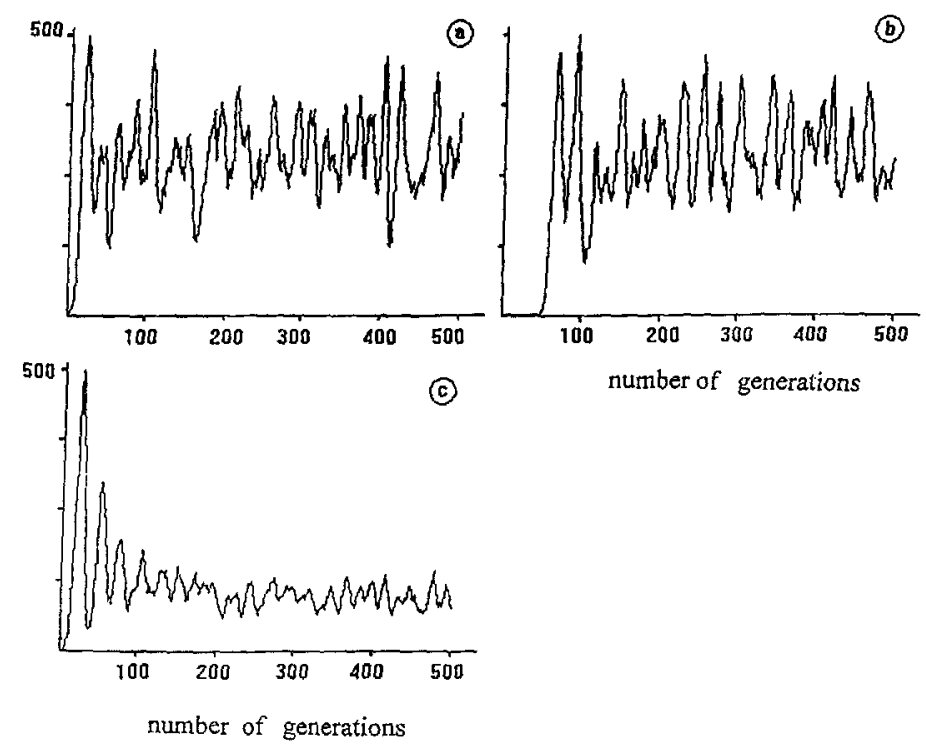

FIGURE 5 Dynamics of heterogeneous clone development in terms of the integer number of proliferating cells in a clone ( $y$ axis) simulated by the model (1)-(3) (taking into accolnt the random character of proliferation/differentiation processes). The parameters of the stable population distribution are: $K_{p}=0.7, C_{p}=20$. Different curves correspond to the different stochastic deviation of the clonal distribution $C_{\mathrm{e}}=C_{\mathrm{p}}(\mathrm{a}) ; 3 C_{\mathrm{p}}(\mathrm{b}) ; 7 C_{\mathrm{p}}(\mathrm{c})$.

and regulated growth up to exponential burst of proliferation (Figure 7). So the high population variability and strong mother-daughter inheritance stipulate the shift of clonal distribution toward the appearance and selection of actively proliferating and insensitive with respect to the action of differentiation factors cells that is to say toward the cells with the cancer phenotype (Figure 8). We called this type of clone development an autoselection phenomenon.

There are some experimental investigations which confirm the results obtained. For instance, a tendency of offspring to resemble their parents more closely than they resemble the mean of population has been demonstrated for the populations of proliferating mammalian cells (Axelrod et al., 1993). The next 


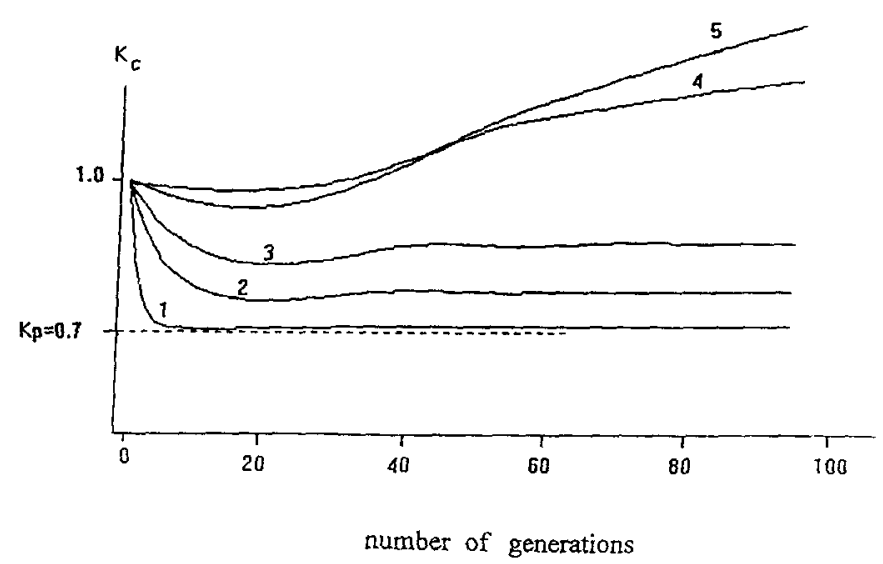

FIGURE 6 Progression of the mean clone value of the mitotic activity index $\boldsymbol{K}_{\mathrm{c}}$. The parameters of the stable population distribution are: $K_{\mathrm{p}}=0.7, C_{\mathrm{p}}=5$. Different curves corresponds to the different stochastic deviation of the clonal distribution $C_{\mathrm{c}}=C_{\mathrm{p}}(1) ; 2 C_{\mathrm{p}}(2)$; $3 C_{\mathrm{p}}(3) ; 4 C_{\mathrm{p}}(4) ; 7 C_{\mathrm{p}}(5)$.
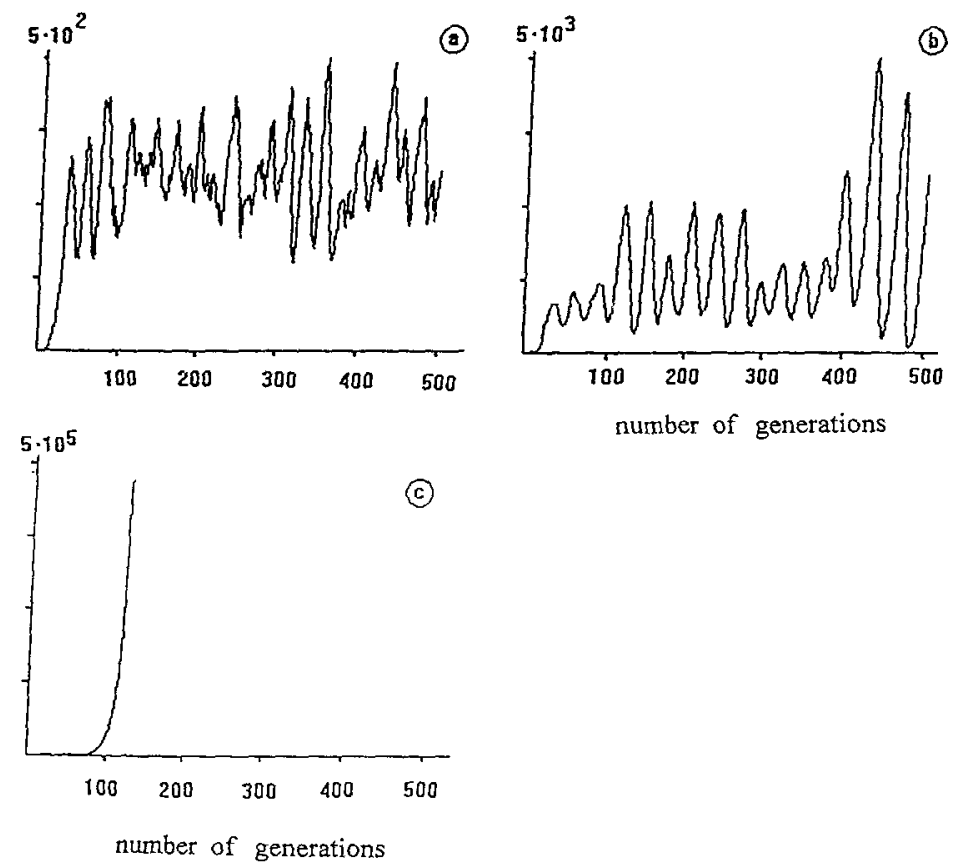

FIGURE 7 Dynamics of heterogeneous clone development in terms of the integer number of proliferating cells in a clone $(y$ axis) simulated by the model (1)-(3) (taking into account the random character of proliferation/differentiation processes). The parameters of the stable population distribution are: $\boldsymbol{K}_{\mathrm{p}}=0.7, C_{\mathrm{p}}=5$. Different curves correspond to the different stochastic deviation of the clonal distribution $C_{\mathrm{c}}=C_{\mathrm{p}}$ (a); $3 C_{\mathrm{p}}$ (b); $7 C_{\mathrm{p}}$ (c).

evidence (although may be being indirect one) - the burst of proliferation often precedes the carcinogenesis; one of the examples - the appearance of focal proliferation (hepatocyte nodules) in the early phase of hepatocarcinogenesis (Ferber, 1991).

\section{DISCUSSION}

During the proliferation (from generation to generation) and differentiation the normal cell clone demonstrates two contrasting ways of its 


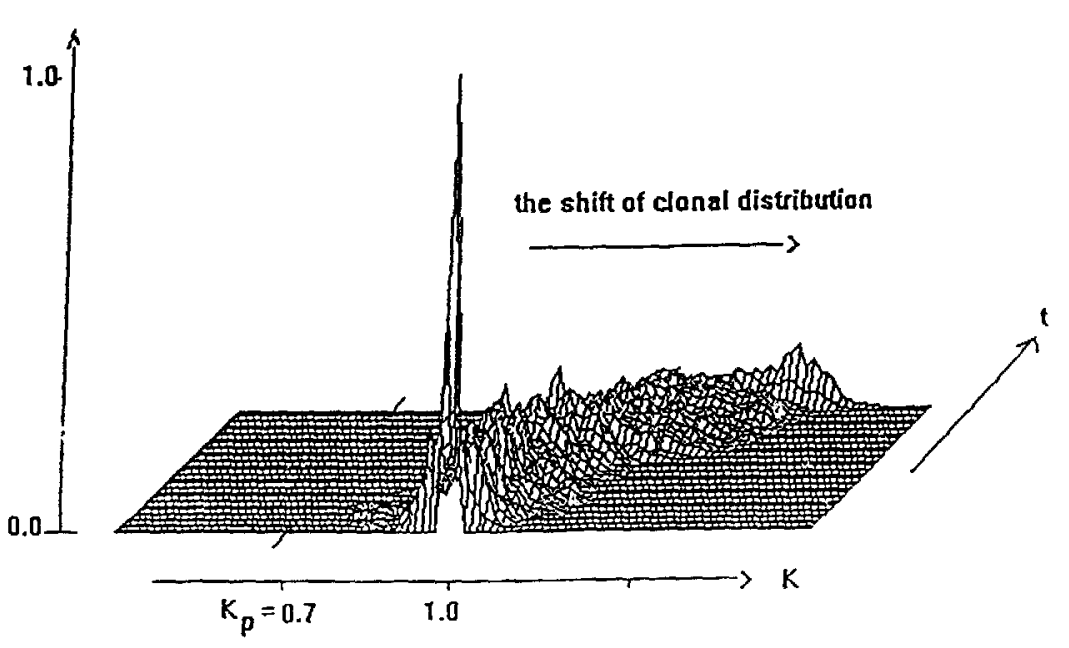

FJGURE 8 Shift of the clonal distribution of the inherent mitotic activity index $(\boldsymbol{K})$ during the evolution (scale $t$ ) of heterogeneous clone. The parameters of the stable population distribution are: $K_{\mathrm{p}}=0.7, C_{\mathrm{p}}=5$. The stochastic deviation of the clonal distribution $C_{\mathrm{c}}>10 C_{\mathrm{p}}$.

development. One of them resulting in the regression of the IMA-index (parameter $k$ ) of the clone cells to a population mean can be considered as a manifestation of the mechanisms ensuring the stability of cell lineage (the genotype stability). Another way leads to the progression of clone towards the appearance and increase of the number of actively proliferating and insensitive to the action of differentiation factors cells (that is to say, the cells with cancer phenotype). This way of clone development can be considered as a possible mechanism of carcinogenesis.

In both cases the evolution of cell clone results from the variability (or heterogeneity) of cell populations, displaying in the diversity of the phenotypic characteristics of the population of genetically identical cells (cell population or cell lineage heterogeneity) and the differences in the inherent mitotic activity between mother and daughter cells (clonal heterogeneity).

In the framework of the model the low level of the clonal heterogeneity of the mitotic activity index is necessary but not sufficient for the emergence of the cells with cancer phenotype. Another important condition which is necessary (and moreover may be sufficient) for the appearance of such cells in the clone is the increase of the population heterogeneity of cells IMA (both conditions together are necessary and sufficient). From our point of view just the weakening of the mechanisms which ensure the stability of cell genotype in more considerable extent causes the cancer shift in cellular composition of the clone than the strong mother-daughter inheritance of the IMA. This result of the model can be tested experimentally: during the early stages of carcinogenesis the growth of the inter-clonal variability of the mitotic activity index (measured in terms of interdivision time or the probability of spontaneous differentiation) has to be considerably higher than the decrease rate of the intraclonal one.

In the framework of our investigation the main mechanism which stipulates the appearance of the cells with cancer phenotype in the normal cell clone under the mentioned above conditions is an autoselection phenomenon. It should be noted that at least at last decade it has been progressively understood that Darwin's principles of heritable variations and natural selection can govern the evolution of all dividing cells in the multicellular organism (Murase, 1996). According to the well known clonal selection theory in oncology the cancer cells appears through the series of successive (point) mutations and natural selection at the level of dividing cells. In the framework of our investigation the cells with cancer phenotype (which is heritable within the variability of mother-daughter correlations!) appears through the series of the permanent nonmutational changes in the proliferating 
cells and through autoselection mechanism retaining for the division and generation of the progeny only that cells which are less sensitive with respect to the action of the differentiation factors. In contrast to the clonal selection theory considering the natural selection as a result of the host selection pressure (in other words this selection is formed as an extraclonal phenomenon) the autoselection mechanism based on the inhibition of the proliferating cells by DF is an intraclonal event.

As it was mentioned above the main (and necessary) link in the proposed mechanism of the carcinogenesis resulting in the emergence of the cells with cancer phenotype is the increase of the phenotypic heterogeneity of the cellular genotype. From our point of view the weakening of the mechanisms which ensure the stability of cell genotype seems to be an early manifestation of genetic instability of the normal cells. The development of this instability may then cause the appearance of genetic mutations with the following progression of the mutated cells. Unfortunately the biochemical mechanisms ensuring the stability of the cell genotype are unknown. Moreover the lack of the strong definition of cell genotype and cell phenotype prevents from the comprehension of the experimental investigations and understanding the genetic or biochemical processes underlying the formation of cell genotype. The discussion of this problems exceeds the bounds of proposed investigation and needs in special consideration.

\section{APPENDIX}

For every mother cell (with IMA-index $\boldsymbol{K}_{\mathrm{m}}$ ) the values of the mitotic activity index of daughter cells $\boldsymbol{K}(\mathrm{j})$ have been calculated by the use of the following stochastic function:

$$
\boldsymbol{K}(\mathrm{j})=\boldsymbol{K}_{\mathrm{p}}+\mathrm{s} \cdot\left(\boldsymbol{K}_{\mathrm{m}}-\boldsymbol{K}_{\mathrm{p}}\right)+\operatorname{sign}(+/-) \cdot \operatorname{Inverf}(\mathrm{j}) / \boldsymbol{C}_{\mathrm{c}}
$$

where $s=\left(1-C_{\mathrm{p}}^{2} / \boldsymbol{C}_{\mathrm{c}}^{2}\right)^{1 / 2}$;

$$
\mathrm{j}=\operatorname{Rnd}(\mathrm{i}) ; \quad \mathrm{i} \in\{0,1\} \text {; }
$$

Inverf(j) - inverted function of the probability integral.

It should be noted that we didn't consider the mutual sister-sister cells correlation.

\section{References}

[1] Axelrod, D. E. and Kuczek, T. (1989). Clonal heterogeneity in populations of normal and tumor cells. Computers Math Applic., 18, 871-881

[2] Axelrod, D. E., Gusev, Y, and Kuczek, T. (1993). Persistence of cell cycle times over many generations as determined by heritability of colony sizes of ras oncogene-transformed and non-transformed cells. Cell Prolif., 26, 235-249.

[3] Dicicco-Bloom, E., Townes-Anerson. E. and Black, I. B (1990). Neuroblast mitosis in dissociated culture: regulation and relationship to differentiation. J.Cell Biol, $\mathbf{1 1 0}$, 2073-2086.

[4] Enrietto, P. J. and Beug, H. (1994). Introduction: oncogenes and differentiation. Seminars in Cancer Biol., 5, 91-94.

[5] Farber, E. (199I). Clonal adaptation as an important phase of hepatocarcinogenesis. Cancer Biochem. Biophys., 12, $157-165$.

[6] Fuchs, E. (1990). Epidermal differentiation: the bare assentials. J. Cell Biol, 111, 2807-2814.

[7] Galton, F. (1889). Natural inheritance. London: Macmillan.

[8] Gamel, J. W. and Axelrod, D. E. (1991). Inheritance and regression toward the mean in heterogeneous cell populations. Cell Prolif., 24, 281-292

[9] Green, M. R. (1989). When the products of oncogenes and anti-oncogenes meet. Cell, 56, 1-3.

[10] Heppner, G. H. (1984). Tumor heterogeneity. Cancer Res, 44, $2259-2265$.

[11] Hurlin, P. J., Foley, K. P., Ayer, D. E., Eisenman, R. N., Hanahan, D. and Arbeit, J. M. (1995). Regulation of Myc and Mad during epidermal differentiation and HPV-associated tumorigenesis. Oncogene, 11, 2487-2501.

[12] Milner, L. A., Bigas, A., Kopan, R., Brashem-Stein, C., Birntstein, I. D. and Martin, D. I. K. (1996). Inhibition of granulocytic differentiation by mNotch1. Proc. Natl. Acad. Sci. USA, 93, 13014-13019.

[13] Murase, M. (1996). Alzheimer's disease as subcellular 'cancer', Progress of Theor. Physics, 95, N1, 1-36.

[14] Riley, P. A. and Hola, M. (1981). Clonal variation in proliferation rate of cultures of GPK cells. Cell \& Tissue Kinet, 14, $489-500$.

[16] Serikov, A. A. and Solyanik, G. 1. (1998). Self-control of proliferation-differentiation processes in cell population dynamics. Physics of the alive, 6, 5-16. 


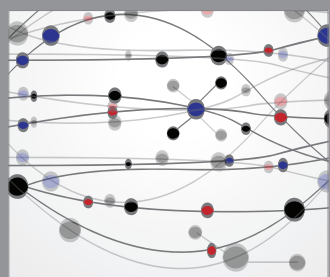

The Scientific World Journal
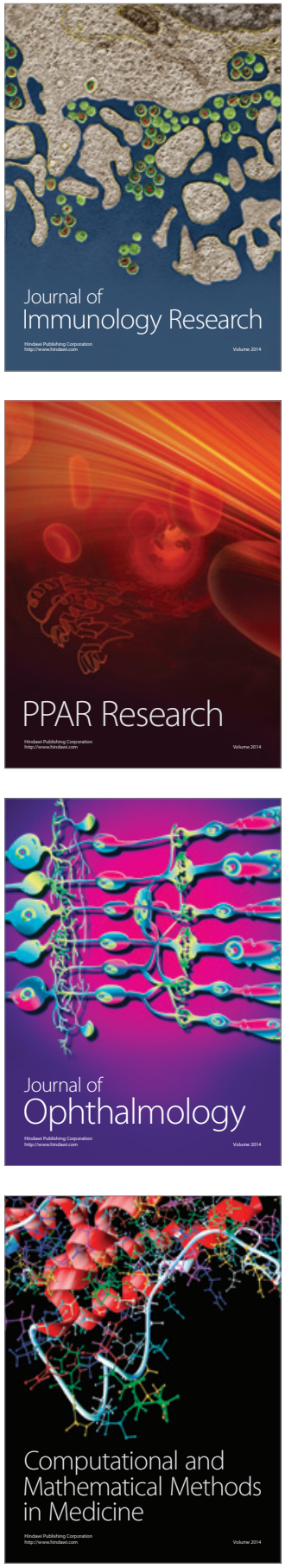

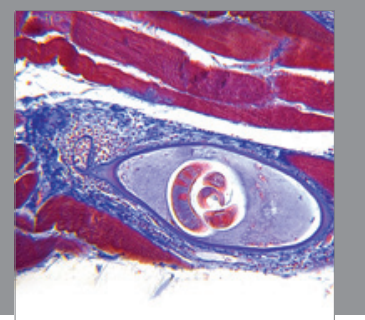

Gastroenterology

Research and Practice
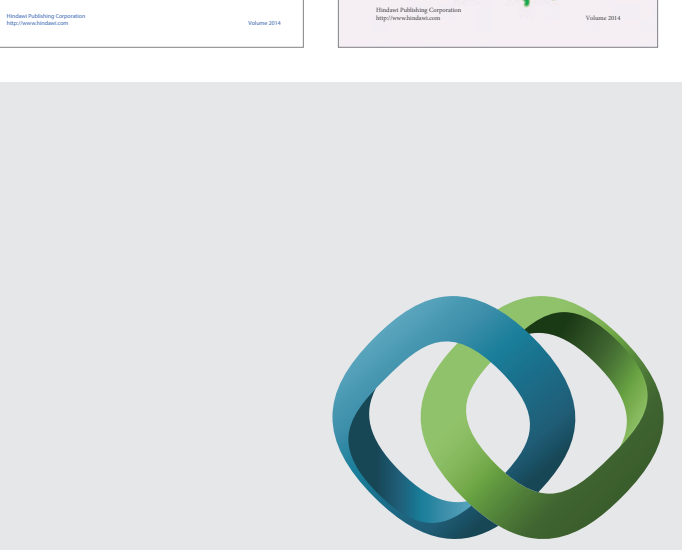

\section{Hindawi}

Submit your manuscripts at

http://www.hindawi.com
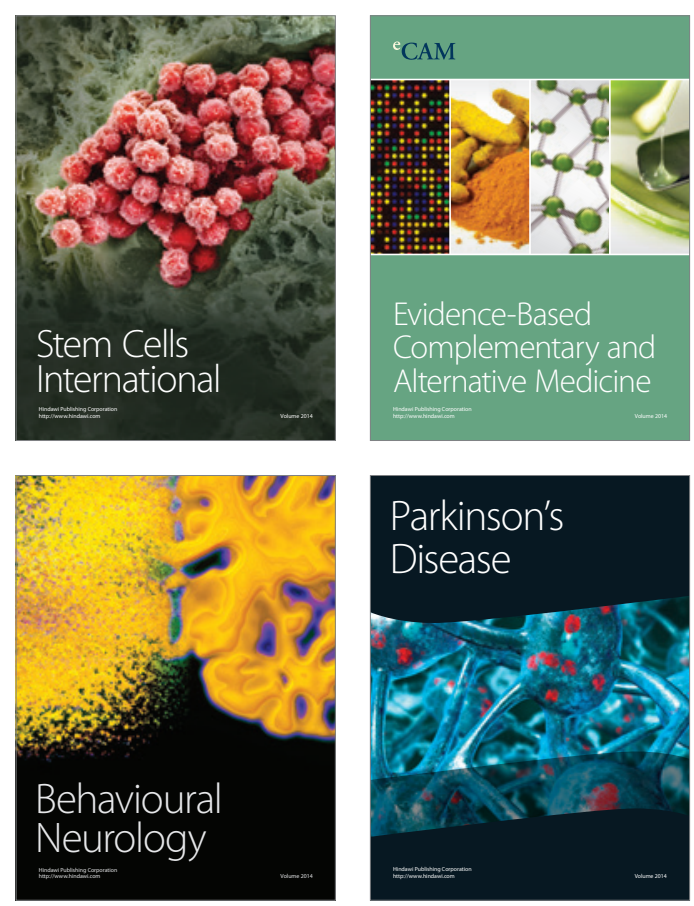

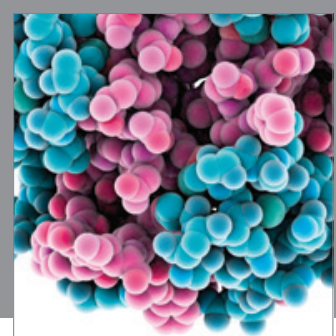

Journal of
Diabetes Research

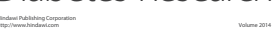

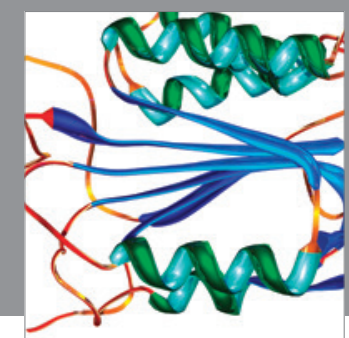

Disease Markers
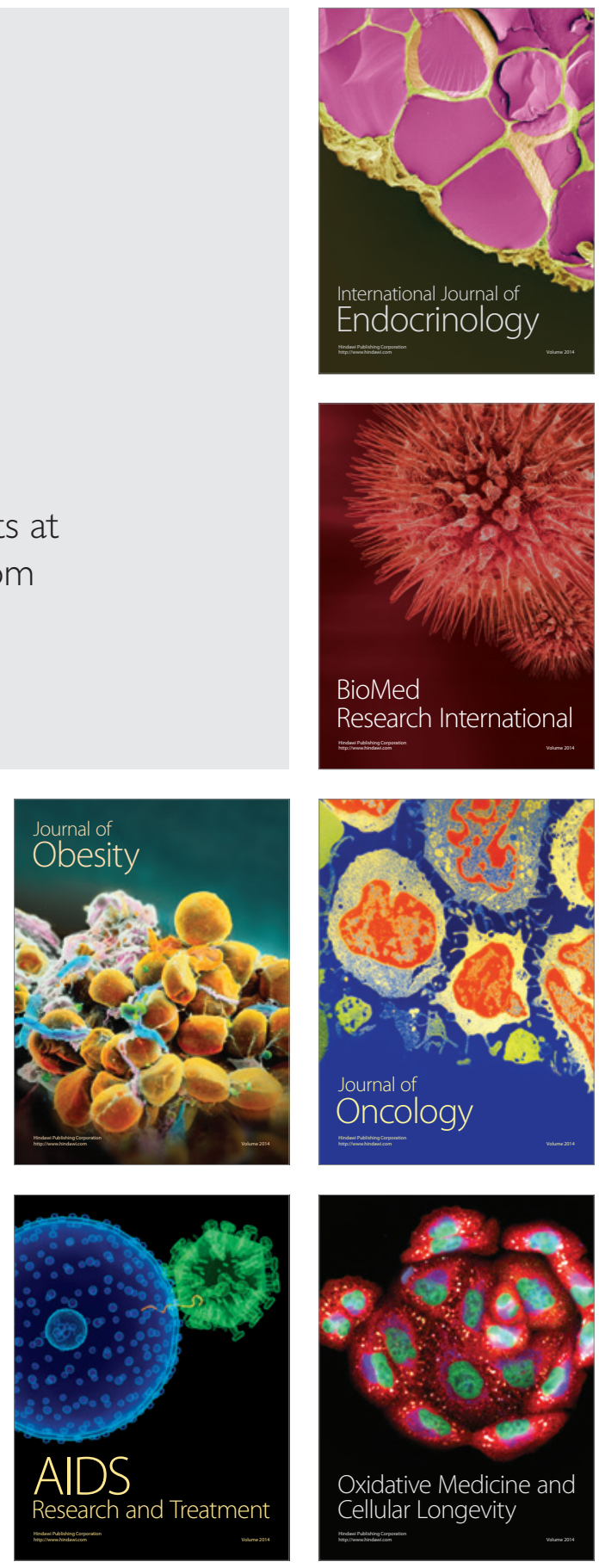